\title{
OPEN Prognostic impact of malignant diseases in idiopathic pulmonary fibrosis
}

\author{
Hong Yeul Lee, Jaeyoung Cho, Nakwon Kwak, Jinwoo Lee, Young Sik Park, Chang-Hoon Lee, \\ Sang-Min Lee, Chul-Gyu Yoo, Young Whan Kim \& Sun Mi Choi ${ }^{\bowtie}$
}

No studies on idiopathic pulmonary fibrosis (IPF) have investigated the prognostic impact of extrapulmonary cancers in patients with IPF. We aimed to determine the prognostic impact of malignancies in patients with IPF. We retrospectively reviewed the medical records of patients diagnosed with IPF between 2001 and 2015. Patients were divided into three groups: IPF without cancer $(n=440)$, IPF with lung cancer $(n=69)$, and IPF with extrapulmonary cancer $(n=70)$. Of the 579 patients with IPF, 139 (24\%) had cancer; the three most common types were lung (11.9\%), gastric (2.4\%), and colorectal (1.9\%). Survival was significantly worse in patients with lung cancer than in those without cancer (hazard ratio $[\mathrm{HR}]=1.83,95 \%$ confidence interval $[\mathrm{Cl}], 1.35-2.48$ ) or those with extrapulmonary cancer $(\mathrm{HR}=1.70,95 \% \mathrm{Cl}, 1.14-2.54)$. The rate of hospitalisation for cancer-related complications was significantly higher in IPF patients with lung cancer than in those with extrapulmonary cancer. The annual rates of decline in percent predicted forced vital capacity and diffusion capacity for carbon monoxide did not differ among the groups. Physicians should pay attention to the development and progression of cancer and its prognostic impact in patients with IPF.

Idiopathic pulmonary fibrosis (IPF) represents the most common type of idiopathic interstitial pneumonia, and it occurs primarily in older adults ${ }^{1}$. Comorbidities are common in IPF patients, and they can negatively influence the quality of life and prognosis ${ }^{2}$. Pulmonary hypertension, chronic obstructive pulmonary disease, ischaemic heart disease, gastro-oesophageal reflux disease, and lung cancer are common comorbidities in IPF patients ${ }^{2}$. IPF and cancer share common risk factors, which include cigarette smoking, viral infections, environmental exposure, diabetes mellitus, and gastro-oesophageal reflux. They also have pathogenetic similarities ${ }^{3,4}$.

Several studies have reported that the incidence of lung cancer in patients with IPF was 11.2-36 cases per 1000 person-years ${ }^{5-7}$. A large cohort study of patients from the United Kingdom found a marked increase in the incidence of lung cancer in patients with IPF when compared with the general population (rate ratio 4.99; 95\% confidence interval [CI], 3.03-8.22), but there was no increase in the incidence of other cancers. Moreover, the association between IPF and lung cancer persisted after adjustment for smoking (rate ratio 4.96; 95\% CI, $3.00-8.18)^{5}$. A retrospective analysis reported that the survival rate was significantly worse in IPF patients with lung cancer than in those without lung cancer (median survival 38.7 months versus 63.9 months) ${ }^{8}$.

Most studies have focused on the prognostic impact of lung cancer in patients with IPF. However, there are limited data on extrapulmonary cancer in patients with IPF. Therefore, our study aimed to investigate the prognostic impact of extrapulmonary cancers in patients with IPF.

\section{Methods}

Study design and subjects. This retrospective cohort study involving patients with IPF was performed at Seoul National University Hospital, a tertiary teaching hospital in Seoul, South Korea. We reviewed all medical records and chest computed tomography images of patients diagnosed with IPF according to European Respiratory Society/American Thoracic Society criteria ${ }^{4,9}$. Patients aged $>18$ years who were newly diagnosed with IPF between January 1, 2001, and December 31, 2015, and followed-up until December 31, 2017, were included. We excluded patients with certain causes of interstitial lung disease, including a history of domestic or occupational environmental exposure, drug toxicity, and connective tissue disease. Patients were divided into three groups: IPF without cancer $(n=440)$, IPF with lung cancer $(n=69)$, and IPF with extrapulmonary cancer $(n=70)$. Patients who were not diagnosed with a malignancy within 5 years before the IPF diagnosis until December 31, 2017, were included in the IPF without cancer group. Patients who were diagnosed with lung cancer or other 


\begin{tabular}{|l|c|l|}
\hline \multirow{2}{*}{ Malignancy } & \multicolumn{2}{|l|}{$\begin{array}{l}\text { IPF cohort } \\
\text { (n=579) }\end{array}$} \\
\cline { 2 - 3 } & Event & $\%$ \\
\hline IPF without cancer & 440 & 76.0 \\
\hline Lung cancer & 69 & 11.9 \\
\hline Gastric cancer & 14 & 2.4 \\
\hline Colorectal cancer & 11 & 1.9 \\
\hline Hepatocellular carcinoma & 11 & 1.9 \\
\hline Prostate cancer & 6 & 1.0 \\
\hline Lymphoma, leukaemia & 6 & 1.0 \\
\hline Pancreatobiliary cancer & 4 & 0.7 \\
\hline Neuroendocrine tumour & 3 & 0.5 \\
\hline Breast cancer & 2 & 0.3 \\
\hline Multiple myeloma & 2 & 0.3 \\
\hline Bladder cancer & 2 & 0.3 \\
\hline Malignancy of undefined primary origin & 2 & 0.3 \\
\hline Renal cell carcinoma & 2 & 0.3 \\
\hline Cervical cancer & 1 & 0.2 \\
\hline Thyroid cancer & 1 & 0.2 \\
\hline Head and neck cancer & 1 & 0.2 \\
\hline Skin cancer & 1 & 0.2 \\
\hline Angiosarcoma & & 0.2 \\
\hline
\end{tabular}

Table 1. Prevalence of malignancies in patients with idiopathic pulmonary fibrosis. IPF idiopathic pulmonary fibrosis.

malignancies within 5 years before the IPF diagnosis until December 31, 2017, were included in the IPF with lung cancer group or IPF with extrapulmonary cancer group, respectively. Cancer survivors usually have regular follow-ups scheduled at least every 5 years after the initial cancer diagnosis. Therefore, we chose this interval in our analysis. To ensure individual privacy, all the data analysed were anonymised and de-identified. The Institutional Review Board of the Seoul National University Hospital waived the requirement for written informed consent and approved this study. (Approval number IRB-H-1803-032-927). It was confirmed that all procedures adhered to the relevant guidelines and regulations. The present study conformed to the STROBE Statement.

Data collection. All medical records were reviewed for IPF diagnosis, sex, comorbidities, smoking history and pack-years, body mass index (BMI), pulmonary function tests including absolute and percent predicted forced vital capacity (FVC), forced expiratory volume in $1 \mathrm{~s}\left(\mathrm{FEV}_{1}\right), \mathrm{FEV}_{1} / \mathrm{FVC}$ ratio, and diffusing capacity for carbon monoxide $\left(\mathrm{D}_{\mathrm{LCO}}\right)$, chest computed tomography, cytological and pathological data, date of IPF diagnosis, date of malignancy diagnosis, number of emergency room (ER) visits and admissions to intensive care unit (ICU), and follow-up duration. The date of death was verified using several medical records and the death registry from the Ministry of the Interior and Safety, South Korea. All the cancers were diagnosed based on the clinical, radiological, and cytological or histopathological examinations. Based on cigarette smoking history, the study population was divided into smokers and non-smokers. Smokers were defined as current and ex-smokers who had smoked more than 100 cigarettes in their lifetime.

Statistical analysis. Continuous baseline characteristics were summarised using the mean and standard deviation or median and interquartile range, whereas categorical characteristics were summarised using frequencies and percentages. Normally distributed quantitative data were compared using the analysis of variance. Quantitative data that were not normally distributed were compared using the Kruskal-Wallis test. Qualitative data were compared using the chi-squared test. The survival curves were estimated using the Kaplan-Meier method and were compared using the log-rank test. The univariate and multivariate Cox regression analyses were used to assess the prognostic impact of malignancies in patients with IPF. The hazard ratios (HRs) and 95\% confidence intervals were estimated. All the analyses were two-tailed, and $p$ values of $<0.05$ were considered statistically significant. SPSS software version 25.0 for Windows (SPSS Inc., Chicago, IL, USA) was used for all statistical analyses.

\section{Results}

Clinical characteristics and prevalence of various cancers in IPF patients. A total of 579 patients newly diagnosed with IPF were included. Of them, 94 patients (16.2\%) had undergone open lung biopsy to confirm the diagnosis of IPF. Among the patients with IPF, 139 (24\%) patients were diagnosed with cancer; the five most common cancers were lung cancer (11.9\%), gastric cancer (2.4\%), colorectal cancer (1.9\%), hepatocellular carcinoma (1.9\%), and prostate cancer (1.0\%). Table 1 shows the prevalence of the various malignancies. The patients were divided into three groups based on the diagnosis of malignancy: IPF without cancer $(n=440$, 


\begin{tabular}{|c|c|c|c|c|}
\hline Variables & $\begin{array}{l}\text { IPF without cancer } \\
(\mathrm{n}=440)\end{array}$ & $\begin{array}{l}\text { IPF with lung cancer } \\
(\mathrm{n}=69)\end{array}$ & $\begin{array}{l}\text { IPF with extrapulmonary cancer } \\
(\mathbf{n}=70)\end{array}$ & $p$ value \\
\hline Age at IPF diagnosis, years & $68.0(61.0-73.0)$ & $69.0(62.0-72.0)$ & $68.0(60.0-73.0)$ & 0.978 \\
\hline Male & $298(67.7 \%)$ & $64(92.8 \%)$ & $57(81.4 \%)$ & $<0.001$ \\
\hline Body mass index, $\mathrm{kg} \cdot \mathrm{m}^{-2}$ & $24.0 \pm 3.1$ & $24.0 \pm 2.8$ & $23.2 \pm 2.9$ & 0.080 \\
\hline \multicolumn{5}{|l|}{ Smoking history } \\
\hline Smokers & $246(58.0 \%)$ & $59(86.8 \%)$ & $53(77.9 \%)$ & $<0.001$ \\
\hline Smoking amount, pack-year & $10.0(0.0-30.0)$ & $40.0(23.5-50.0)$ & $20.0(10.0-40.0)$ & $<0.001$ \\
\hline \multicolumn{5}{|l|}{ Comorbidities } \\
\hline History of HT & $148(33.6 \%)$ & $26(37.7 \%)$ & $22(31.4 \%)$ & 0.725 \\
\hline History of DM & $121(27.5 \%)$ & $13(18.8 \%)$ & $20(28.6 \%)$ & 0.294 \\
\hline History of TB & $74(16.8 \%)$ & $10(14.5 \%)$ & $12(17.1 \%)$ & 0.882 \\
\hline \multicolumn{5}{|l|}{ Lung function } \\
\hline FVC, L & $2.40(1.92-3.01)$ & $2.94(2.53-3.44)$ & $2.78(2.41-3.31)$ & $<0.001$ \\
\hline FVC, $\%$ pred & $74.0(61.0-87.0)$ & $78.0(72.0-93.0)$ & $82.0(69.0-96.0)$ & 0.002 \\
\hline $\mathrm{FEV}_{1}, \mathrm{~L}$ & $1.98(1.61-2.42)$ & $2.35(2.07-2.65)$ & $2.24(2.00-2.61)$ & $<0.001$ \\
\hline $\mathrm{FEV}_{1}, \%$ pred & $88.0(74.0-103.0)$ & $88.0(82.0-103.0)$ & $94.0(76.0-114.0)$ & 0.079 \\
\hline $\mathrm{FEV}_{1} / \mathrm{FVC}, \%$ & $83.0(77.5-87.0)$ & $79.0(74.0-84.0)$ & $82.0(75.0-86.0)$ & $<0.001$ \\
\hline $\mathrm{D}_{\mathrm{LCO}}, \mathrm{mL} / \mathrm{mm} \mathrm{Hg} / \mathrm{min}$ & $10.3(7.8-12.7)$ & $12.5(10.3-14.7)$ & $11.5(7.9-14.3)$ & $<0.001$ \\
\hline $\mathrm{D}_{\mathrm{LCO}}, \%$ pred & $59.5(48.0-75.0)$ & $68.0(56.0-84.0)$ & $66.0(50.0-86.0)$ & 0.005 \\
\hline GAP score & $3.1 \pm 1.4$ & $3.0 \pm 1.2$ & $3.0 \pm 1.2$ & 0.302 \\
\hline
\end{tabular}

Table 2. Baseline characteristics of the study population. Data are presented as $\mathrm{n}(\%)$, mean \pm standard deviation, or median (interquartile range). $D_{L C O}$ diffusing capacity of the lung for carbon monoxide, $D M$ diabetes mellitus, $F E V_{1}$ forced expiratory volume in $1 \mathrm{~s}, F V C$ forced vital capacity, GAP score gender, age, and physiology score, $H T$ hypertension; IPF idiopathic pulmonary fibrosis, TB pulmonary tuberculosis, $\%$ pred percent predicted.

$76 \%)$, IPF with lung cancer ( $n=69,11.9 \%)$, and IPF with extrapulmonary cancer $(n=70,12.1 \%)$. The baseline clinical characteristics of IPF patients are shown in Table 2. For all patients with IPF, the median age at the time of IPF diagnosis was 68 years; $72.4 \%$ were men, mean BMI was $23.9,63.9 \%$ were smokers, $33.9 \%$ had a history of hypertension, $26.6 \%$ had a history of diabetes mellitus, $16.6 \%$ had a history of tuberculosis, and the mean gender, age, and physiology score was $3.1 \pm 1.4$ points. Cancer comorbidities in IPF patients were more common in men and smokers. Patients with lung cancer had higher percent predicted FVC and predicted $\mathrm{D}_{\mathrm{LCO}}$ as well as a lower $\mathrm{FEV}_{1} / \mathrm{FVC}$ ratio than those without cancer. Among the IPF patients with lung cancer, 2 patients $(2.9 \%)$ were diagnosed with cancer before the IPF diagnosis, whereas 67 patients $(97.1 \%)$ were diagnosed with cancer concomitantly $(34.8 \%)$ or after $(62.3 \%)$ the IPF diagnosis. The initial stages of lung cancer were as follows: 21 patients (30.4\%) in stage I; 10 patients (14.5\%) in stage II; 19 patients $(27.5 \%)$ in stage III; and 19 patients $(27.5 \%)$ in stage IV. The histologic types of lung cancer were squamous carcinoma in 24 patients (34.8\%), adenocarcinoma in 23 patients (33.8\%), unclassified non-small-cell lung cancer (NSCLC) in 12 patients (17.4\%), smallcell lung cancer (SCLC) in 7 patients (10.1\%), and large-cell lung cancer in 3 patients (4.3\%). Among the IPF patients with extrapulmonary cancer, 20 patients $(28.6 \%)$ were diagnosed with cancer before the IPF diagnosis, whereas 50 patients $(71.4 \%)$ were diagnosed with cancer concomitantly $(25.7 \%)$ or after (45.7\%) the IPF diagnosis. Among the IPF patients with lung cancer stage I, 12 (57.1\%), 8 (38.1\%), and 1 (4.8\%) were treated with surgery, radiotherapy, and chemotherapy, respectively. Further details on the first-line treatments stratified by lung-cancer stage are provided in Supplementary Table S1.

Prognostic impact of malignant diseases in IPF patients. Among the 579 patients with IPF, 382 (66.0\%) died during the study period and the median survival time was 4.89 years (95\% CI, 4.29-5.49). Among the IPF patients without cancer, 279 (63.4\%) patients died and the median survival time was 5.45 years (95\% CI, 4.73-6.18). Among the IPF patients with lung cancer, 57 (82.6\%) patients died, and the median survival time was 2.93 years (95\% CI, 2.30-3.56). Among the IPF patients with extrapulmonary cancer, 46 (65.7\%) patients died, and the median survival time was 4.84 years (95\% CI, 3.13-6.54). The 1-year, 5-year, and 10-year survival rates were $92.0 \%, 51.7 \%$, and $27.1 \%$, respectively, in the IPF group without cancer, $81.2 \%, 35.7 \%$, and $12.2 \%$, respectively, in the IPF group with lung cancer, and 92.9\%, 49.0\%, and 19.6\%, respectively, in the IPF group with extrapulmonary cancer. Figure 1a shows the Kaplan-Meier estimates of the overall survival. There was a significant difference between the survival rates of the groups $(p=0.001)$. The log-rank test showed a significantly worse survival in the IPF group with lung cancer than in the group without cancer $(p<0.001)$ or with extrapulmonary cancer $(p=0.021)$. No significant difference was observed between the survival rates of IPF patients without cancer and those with extrapulmonary cancer $(p=0.726)$. The patients were divided into two groups based on the stage of cancer: early (stage I to IIIa NSCLC; limited-stage SCLC) and advanced (stage IIIb to IV NSCLC; extensive-stage SCLC). Further analysis revealed that patients with advanced-stage lung cancer had a significantly worse survival rate than those in the other groups $(p<0.001)$ (Fig. 1b). IPF patients with early-stage 
a

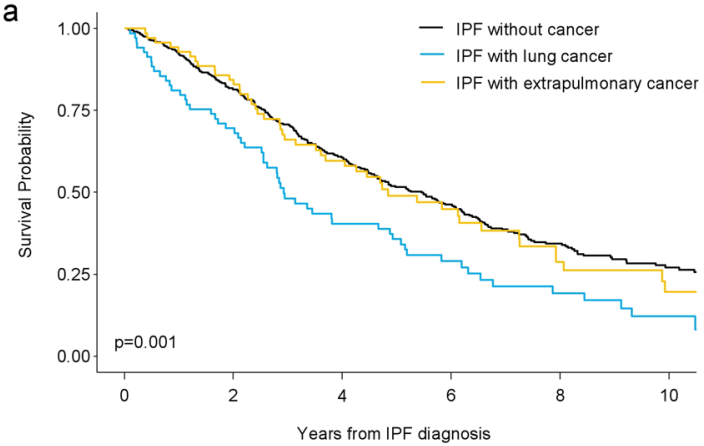

C

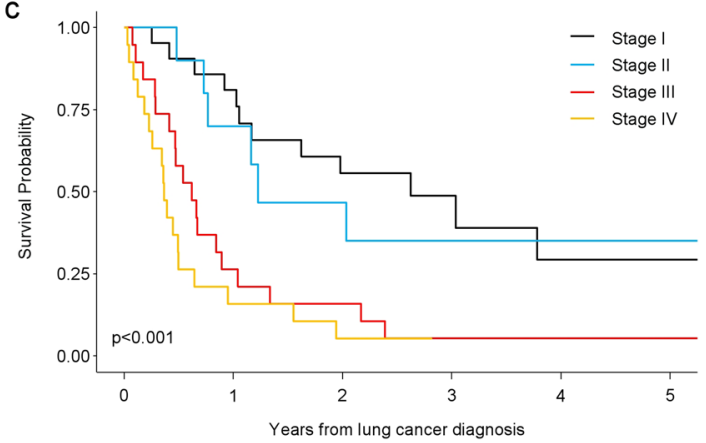

b

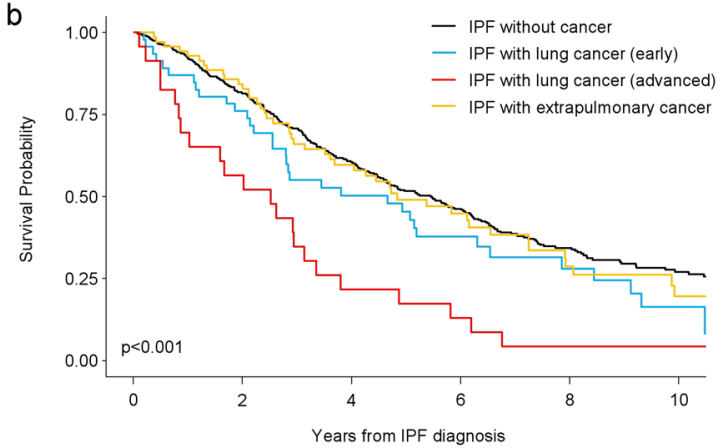

d

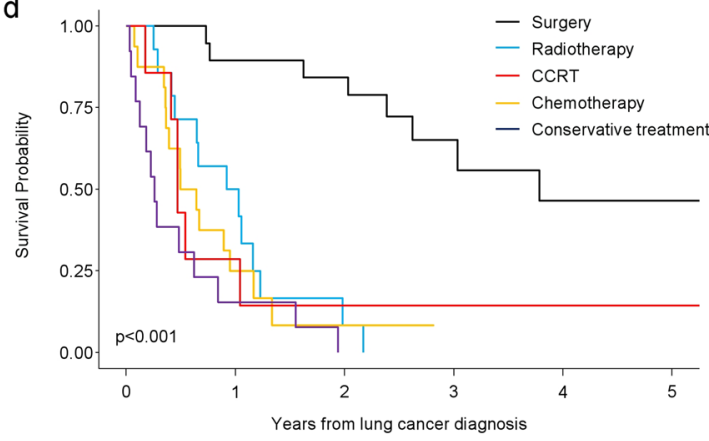

Figure 1. Kaplan-Meier curves for survival in patients with idiopathic pulmonary fibrosis (IPF) stratified by cancer status. (a) The IPF patients with lung cancer group are further classified into the early and advanced groups. (b) Subgroup analysis for survival in IPF patients with lung cancer according to the cancer stage at diagnosis (c) and first-line treatment after lung cancer diagnosis (d). IPF idiopathic pulmonary fibrosis.

lung cancer did not have a worse survival rate than those without cancer $(p=0.075)$ or those with extrapulmonary cancer $(p=0.246)$. Among the IPF patients with lung cancer, the survival rate was worse in those with more advanced stages at diagnosis (Fig. $1 c ; p<0.001$ ), and the surgery group showed the most favourable survival (Fig. 1d; $p<0.001$ ).

The univariate Cox regression analysis showed that lung cancer comorbidities, male sex, older age at the time of IPF diagnosis, lower BMI, percent predicted FVC lower than $80 \%$, and percent predicted $\mathrm{D}_{\mathrm{LCO}}$ lower than $80 \%$ were associated with worse survival in patients with IPF. In the multivariate analysis, which included variables with a $p$ value of 0.20 or less in the univariate analysis, all of these factors remained significantly associated with worse survival in patients with IPF (Table 3). In the multivariate analysis, IPF patients with lung cancer had significantly worse survival than those without cancer $(\mathrm{HR}=1.83 ; 95 \% \mathrm{CI}, 1.35-2.48 ; p<0.001)$ or those with extrapulmonary cancer $(\mathrm{HR}=1.70 ; 95 \% \mathrm{CI}, 1.14-2.54 ; p=0.009)$. The survival rates of IPF patients without cancer and those with extrapulmonary cancer were not significantly different (HR=1.08; 95\% CI, 0.78-1.48; $p=0.652$ ). Among IPF patients with lung cancer, Cox regression analysis adjusted for age, sex, BMI, percent predicted $\mathrm{FVC}$, and percent predicted $\mathrm{D}_{\mathrm{LCO}}$, revealed that the survival rate was significantly worse in those with more advanced stages at diagnosis, and those who received first-line treatment with surgery and concurrent chemoradiation therapy showed a more favourable survival rate than those who received conservative treatment (Table 4).

The overall rates of respiratory and non-respiratory hospitalisation among IPF patients per 100 personyears were 30.2 (95\% CI, 25.4-35.0) and 21.6 (95\% CI, 17.5-25.7), respectively. The rates of all-cause and nonrespiratory hospitalisation were significantly higher among IPF patients with cancer than in those without cancer ( $p<0.001$ and $p<0.001$, respectively) (Supplementary Table S2). The rate of respiratory hospitalisation was highest among IPF patients with lung cancer. The rate of hospitalisation for cancer-related complications was significantly higher in IPF patients with lung cancer than in those with extrapulmonary cancer $(p=0.011)$. The overall rates of ER visits and ICU admissions per 100 person-years were 39.3 (95\% CI, 33.2-45.4) and 9.7 (95\% CI, 7.4-12.0), respectively, in IPF patients. The rates of ER visits and ICU admissions were significantly higher among IPF patients with cancer than those without cancer $(p<0.001$ and $p<0.001$, respectively) (Fig. 2). In IPF patients without cancer, the rates of ER visits and ICU admissions per 100 person-years were $29.8(95 \%$ CI, 24.1-35.5) and 6.0 (95\% CI, 4.1-7.9), respectively. In patients with lung cancer, the rates of ER visits and ICU admissions per 100 person-years were 77.8 (95\% CI, 50.7-104.8) and 22.9 (95\% CI, 13.2-32.5), respectively. The rates of ER visits and ICU admissions per 100 person-years in patients with extrapulmonary cancer were 60.8 (95\% CI, 38.8-82.7) and 20.0 (95\% CI, 9.5-30.5), respectively. However, the rates of ER visits and ICU admissions of IPF patients with lung cancer and those with extrapulmonary cancer were not significantly different ( $p=0.389$ and $p=0.826$, respectively). Of the IPF patients, $54.0 \%, 27.3 \%$, and $11.0 \%$ were admitted to the ICU for respiratory causes, postoperative management, and cardiovascular causes, respectively. Of 111 IPF patients who died in the hospital, 64 patients (57.7\%) had end-of-life discussions with their physicians and refused ICU 


\begin{tabular}{|c|c|c|c|c|c|}
\hline \multirow[b]{2}{*}{ Variables } & \multirow[b]{2}{*}{ No. of patients } & \multicolumn{2}{|c|}{ Univariate analysis } & \multicolumn{2}{|c|}{ Multivariate analysis } \\
\hline & & HR $(95 \%$ CI) & $p$ value & HR $(95 \% \mathrm{CI})$ & $p$ value \\
\hline \multicolumn{6}{|l|}{ Malignancy } \\
\hline IPF without cancer & 440 & Ref & & Ref & \\
\hline IPF with lung cancer & 69 & $1.69(1.27-2.25)$ & $<0.001$ & $1.83(1.35-2.48)$ & $<0.001$ \\
\hline IPF with extrapulmonary cancer & 70 & $1.06(0.77-1.44)$ & 0.726 & $1.08(0.78-1.48)$ & 0.652 \\
\hline \multicolumn{6}{|l|}{ Sex } \\
\hline Female & 160 & Ref & & Ref & \\
\hline Male & 419 & $1.75(1.37-2.24)$ & $<0.001$ & $1.65(1.26-2.15)$ & $<0.001$ \\
\hline \multicolumn{6}{|l|}{ Age at IPF diagnosis, years } \\
\hline$<65$ & 217 & Ref & & Ref & \\
\hline$\geq 65$ & 362 & $2.41(1.93-3.02)$ & $<0.001$ & $2.61(2.06-3.31)$ & $<0.001$ \\
\hline \multicolumn{6}{|l|}{ Body mass index, $\mathrm{kg} \cdot \mathrm{m}^{-2}$} \\
\hline$<18.5$ & 26 & Ref & & Ref & \\
\hline$\geq 18.5-<25$ & 349 & $0.30(0.20-0.47)$ & $<0.001$ & $0.39(0.25-0.60)$ & $<0.001$ \\
\hline$\geq 25$ & 204 & $0.23(0.15-0.35)$ & $<0.001$ & $0.30(0.19-0.47)$ & $<0.001$ \\
\hline \multicolumn{6}{|l|}{ Smoking, pack-years } \\
\hline$<20$ & 255 & Ref & & & \\
\hline$\geq 20$ & 263 & $1.14(0.92-1.42)$ & 0.226 & & \\
\hline \multicolumn{6}{|l|}{ Hypertension } \\
\hline None & 383 & Ref & & & \\
\hline Present & 196 & $0.96(0.78-1.19)$ & 0.707 & & \\
\hline \multicolumn{6}{|l|}{ Diabetes mellitus } \\
\hline None & 425 & Ref & & Ref & \\
\hline Present & 154 & $1.22(0.97-1.52)$ & 0.084 & $1.06(0.84-1.34)$ & 0.609 \\
\hline \multicolumn{6}{|l|}{ FVC, \% pred } \\
\hline$\geq 80$ & 245 & Ref & & Ref & \\
\hline$<80$ & 333 & $1.64(1.33-2.02)$ & $<0.001$ & $1.51(1.16-1.96)$ & 0.002 \\
\hline \multicolumn{6}{|l|}{$F E V_{1}, \%$ pred } \\
\hline$\geq 80$ & 387 & Ref & & Ref & \\
\hline$<80$ & 191 & $1.20(0.97-1.48)$ & 0.089 & $1.06(0.82-1.38)$ & 0.656 \\
\hline \multicolumn{6}{|l|}{$F E V_{1} / F V C, \%$} \\
\hline$\geq 70$ & 541 & Ref & & & \\
\hline$<70$ & 37 & $0.80(0.52-1.23)$ & 0.313 & & \\
\hline \multicolumn{6}{|l|}{$D_{L C O}, \%$ pred } \\
\hline$\geq 80$ & 126 & Ref & & Ref & \\
\hline$<80$ & 439 & $1.36(1.05-1.76)$ & 0.021 & $1.68(1.27-2.22)$ & 0.001 \\
\hline
\end{tabular}

Table 3. Univariate and multivariate Cox regression analysis of the prognostic factors associated with survival in patients with idiopathic pulmonary fibrosis. $D_{L C O}$ diffusing capacity of the lung for carbon monoxide, $F E V_{1}$ forced expiratory volume in $1 \mathrm{~s}, F V C$ forced vital capacity, $H R$ hazard ratio, IPF idiopathic pulmonary fibrosis, $\%$ pred percent predicted. All variables with a $p$ value of 0.20 or less in the univariate analysis were included in the multivariate analysis.

admission. There was no significant difference between the proportions of patients who refused ICU admission among the groups $(p=0.189)$. The overall ICU mortality rate was $24.2 \%$ in patients with IPF; there was no significant difference in the ICU mortality rates of the groups $(p=0.256)$.

Lung function. Figure 3 shows the annual rates of change in FVC and $\mathrm{D}_{\mathrm{LCO}}$. In IPF patients without cancer, the annual rates of absolute change in percent predicted FVC, FVC (ml/year), and percent predicted $\mathrm{D}_{\mathrm{LCO}}$ from the baseline were $-5.2 \pm 11.0 \%$ per year, $-201.9 \pm 36.4 \mathrm{ml}$ per year, and $-7.4 \pm 14.1 \%$ per year, respectively. In patients with lung cancer, the rates were $-6.7 \pm 12.6 \%$ per year, $-264.7 \pm 47.0 \mathrm{ml}$ per year, and $-8.5 \pm 16.7 \%$ per year, respectively. In patients with extrapulmonary cancer, the rates were $-5.5 \pm 12.2 \%$ per year, $-212.7 \pm 48.6 \mathrm{ml}$ per year, and $-7.1 \pm 9.8 \%$ per year, respectively. The annual rates of absolute change in percent predicted FVC, FVC (ml/year), and percent predicted $\mathrm{D}_{\mathrm{LCO}}$ from the baseline did not differ significantly among the groups $(p=0.672, p=0.544$, and $p=0.854$, respectively). 


\begin{tabular}{|l|l|l|r|}
\hline Variables & No. of patients & Adjusted HR $\mathbf{~}^{*} \mathbf{9} \%$ CI $)$ & $p$ value \\
\hline Lung cancer stage at diagnosis & 21 & Ref & \\
\hline Stage I & 10 & $1.23(0.49-3.10)$ & 0.657 \\
\hline Stage II & 19 & $3.79(1.73-8.27)$ & 0.001 \\
\hline Stage III & 19 & $5.17(2.31-11.5)$ & $<0.001$ \\
\hline Stage IV & \multicolumn{4}{l|}{} \\
\hline First-line treatment of cancer & 13 & Ref & \\
\hline Conservative treatment & 19 & $0.09(0.03-0.23)$ & $<0.001$ \\
\hline Surgery & 19 & $0.56(0.25-1.24)$ & 0.149 \\
\hline Radiotherapy & 14 & $0.30(0.10-0.91)$ & 0.034 \\
\hline CCRT & 7 & $0.69(0.30-1.59)$ & 0.385 \\
\hline Chemotherapy & 16 & &
\end{tabular}

Table 4. Cox regression analysis of the prognostic factors associated with survival in lung cancer patients with idiopathic pulmonary fibrosis. CCRT concurrent chemoradiation therapy, $H R$ hazard ratio. *Adjusted for sex, age, body mass index, forced vital capacity (percent predicted), and diffusing capacity of the lung for carbon monoxide (percent predicted).
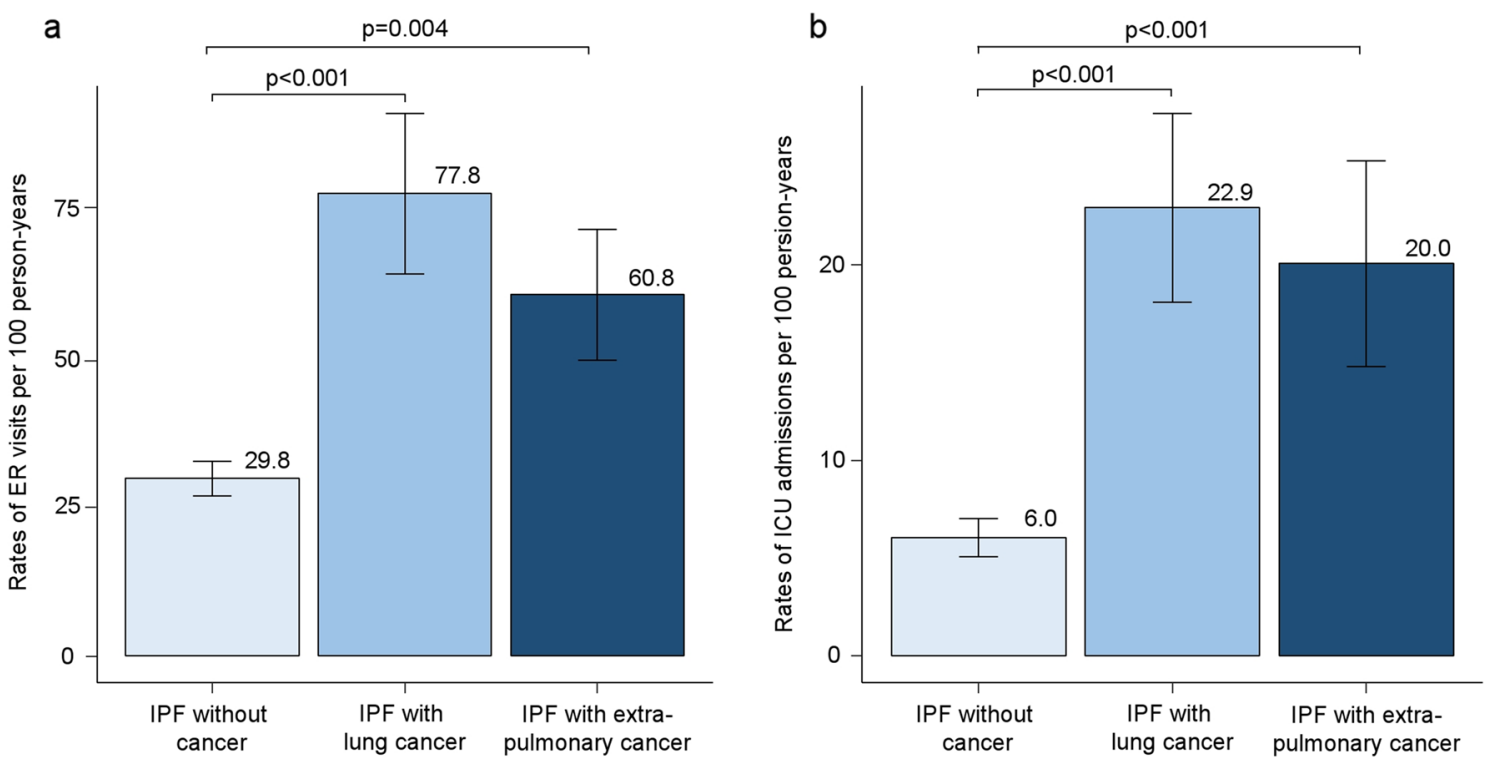

Figure 2. Rates of emergency room visit and admission to the intensive care unit per 100 person-years. (a) The differences in the rates of emergency room (ER) visits per 100 person-years among the groups. I bars indicate the standard error. (b) The differences in the rates of admission to the intensive care unit (ICU) per 100 personyears among the groups. I bars indicate the standard error. IPF idiopathic pulmonary fibrosis.

\section{Discussion}

The present retrospective longitudinal cohort study revealed that the prevalence of cancer in IPF patients was $24 \%$ and that of lung cancer was $11.9 \%$. Lung cancer was the most common cancer type among IPF patients. Lung cancer comorbidity was an independent prognostic factor for poor survival in patients with IPF, regardless of age, sex, BMI, percent predicted FVC, and percent predicted $\mathrm{D}_{\mathrm{LCO}}$. However, extrapulmonary cancer comorbidity was not associated with poor survival in patients with IPF. The overall rates of ER visits, all-cause hospitalisation, and ICU admission were significantly higher among IPF patients with cancer than those without cancer. However, the rate of hospitalisation for cancer-related complications was significantly higher in IPF patients with lung cancer than in those with extrapulmonary cancer. The annual rates of absolute change in FVC and $\mathrm{D}_{\mathrm{LCO}}$ from the baseline did not differ significantly among the groups.

Previous studies have shown that lung cancer in IPF patients was more common in men and smokers. IPF patients with lung cancer had higher FVC and lower $\mathrm{FEV}_{1} / \mathrm{FVC}$ than those without cancer ${ }^{6,8,10,11}$. These results are consistent with those of this study. Cigarette smoking has been reported as the major cause of emphysema and various cancers ${ }^{12,13}$, and patients with combined pulmonary fibrosis and emphysema had significantly higher $\mathrm{FVC}$ and lower $\mathrm{FEV}_{1} / \mathrm{FVC}$ than those with pulmonary fibrosis alone $\mathrm{e}^{11,14}$. These are similar to our findings on IPF patients with cancer. 
a

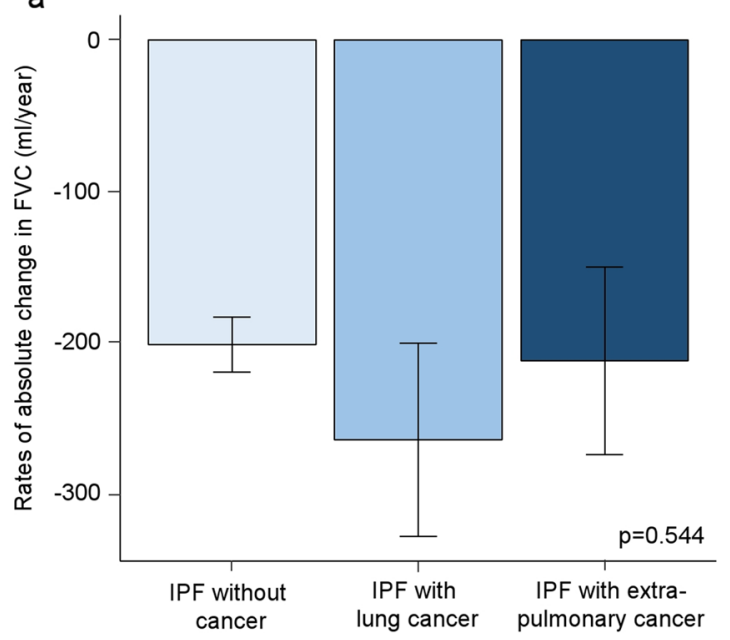

b

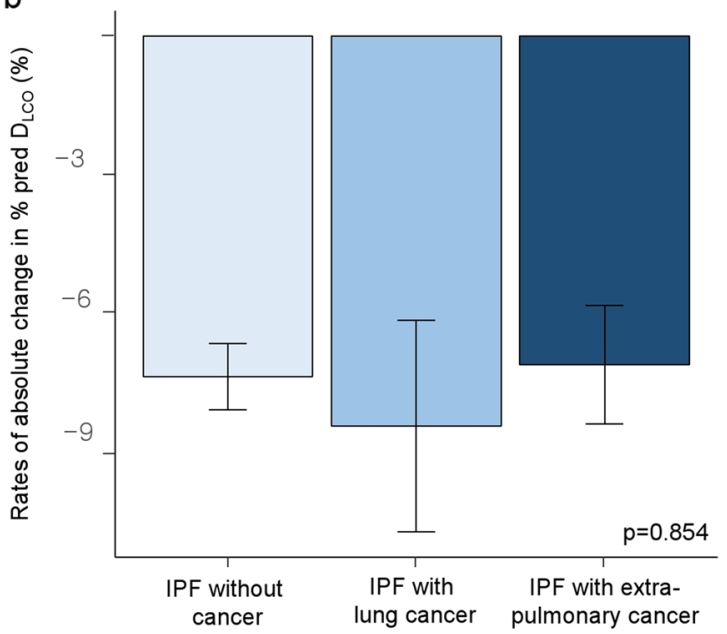

Figure 3. Annual rates of absolute change from the baseline in FVC and $\mathrm{D}_{\mathrm{LCO}}$. (a) The differences in the annual rates of absolute change in FVC among the groups. I bars indicate the standard error. (b) The differences in the annual rates of absolute change in percent predicted $\mathrm{D}_{\mathrm{LCO}}$ among the groups. I bars indicate the standard error. $I P F$ idiopathic pulmonary fibrosis, $F V C$ forced vital capacity, $D_{L C O}$ diffusing capacity of the lung for carbon monoxide, \% pred percent predicted.

Our analysis showed that the prevalence of cancer among IPF patients was $24 \%$, and lung cancer was the most common cancer $(11.9 \%)$, followed by gastric cancer $(2.4 \%)$, colorectal cancer $(1.9 \%)$, hepatocellular carcinoma (1.9\%), and prostate cancer $(1.0 \%)$. There are some variations in the published prevalence of lung cancer in patients with IPF, ranging from $4.8 \%$ to $48 \%$. These values are significantly higher than those in patients without IPF $(2.0 \% \text { to } 6.4 \%)^{15}$. A recent meta-analysis, which included 131,947 patients with IPF from 35 studies, reported that the overall lung cancer prevalence was $13.5 \%(95 \% \text { CI, 10.4-17.4 })^{16}$. However, data on extrapulmonary cancers in patients with IPF are limited. An observational retrospective long-term analysis of IPF patients who were screened for comorbidities reported that out of 272 patients with IPF, 60 (22.1\%) had cancer, $42(15.4 \%)$ had lung cancer, and 18 (6.6\%) had extrapulmonary cancers ${ }^{17}$. These results are similar to ours. The mechanisms of the development of cancer in IPF patients remain unclear. Some mechanisms have been proposed for the association between IPF and cancer. IPF and cancer share common risk factors, especially cigarette smoking ${ }^{3}$. Cigarette smoking is a well-known major risk factor for the development of various cancers ${ }^{12}$. The International Agency for Research on Cancer report shows that there is sufficient evidence on the carcinogenicity of cigarette smoking in humans ${ }^{18}$. Moreover, IPF and cancer have pathogenetic similarities, such as epigenetic and genetic abnormalities, abnormal activation of specific signal transduction pathways, and altered cell-to-cell communications ${ }^{3}$. However, due to the small sample size and the design of this study, the association between IPF and cancer development could not be fully analysed and explained. Further well-designed, population-based studies are needed to compare the risks of various cancers in patients with and without IPF.

Previous studies have reported several predictors of poor survival in IPF at baseline, which include older age, male sex, worse dyspnoea, lower FVC, lower $\mathrm{D}_{\mathrm{LCO}}$, lower BMI, need for supplemental oxygen, greater extent of fibrosis on chest computed tomography, and shorter distance on the six-minute walk test ${ }^{19-23}$. Similarly, the multivariate Cox proportional hazard model in the present study showed that older age, male sex, lower BMI, percent predicted FVC lower than $80 \%$, and percent predicted $\mathrm{D}_{\mathrm{LCO}}$ lower than $80 \%$ at baseline were associated with poor survival in patients with IPF. In addition, our results showed that IPF patients with lung cancer had a significantly poor survival than those without cancer or those with extrapulmonary cancer, even after multivariable adjustment for other known predictors of poor survival. Several studies have reported that the presence of lung cancer among IPF patients was an independent predictor of poor survival ${ }^{8,10,17}$. A previous study from Italy reported that survival in IPF patients was worse in those with than those without lung cancer $(\mathrm{HR}=5.0$; $95 \% \mathrm{CI}$, 2.91-8.57; $p<0.001)$. Causes of death in this study were respiratory failure (43\%), lung cancer treatment-related complications $(17 \%)$, and progression of lung cancer $(13 \%)^{8}$. In a previous retrospective long-term analysis of IPF patients, lung cancer comorbidity was associated with poor survival ( $\mathrm{HR}=2.92 ; 95 \% \mathrm{CI}, 1.62-5.28 ; p<0.001)$, while extrapulmonary cancer comorbidity had no impact on survival in patients with IPF $(\mathrm{HR}=1.15 ; 95 \% \mathrm{CI}$, $0.57-2.31 ; p=0.702)$; this was similar to our results ${ }^{17}$. In this study, IPF patients with early-stage lung cancer and those without cancer or with extrapulmonary cancer had comparable survival rates. Additionally, among IPF patients with lung cancer, the survival rate was worse in those with more advanced stages at diagnosis, and those who received first-line treatment with surgery showed the most favourable survival. Currently, there are no recommendations on how to manage IPF patients with lung cancer. A recent study showed that surgery was effective for lung cancer in patients with IPF, which was consistent with our results ${ }^{24}$. Accordingly, active treatment, such as surgical treatment, may be effective for IPF patients with early-stage lung cancer. The findings from this study add to the evidence that lung cancer comorbidity is an independent prognostic factor for poor 
survival in patients with IPF, while extrapulmonary cancer comorbidity is not associated with poor survival in patients with IPF.

The data on emergency department visits, ICU admissions, and annual change in lung function in IPF patients with cancer are limited in the literature. In this study, overall rates of ER visits and ICU admissions were significantly higher among IPF patients with cancer than those without cancer, while annual rates of absolute change in $\mathrm{FVC}$ and $\mathrm{D}_{\mathrm{LCO}}$ from the baseline did not differ significantly among the groups. A previous systematic review reported that the rates of emergency department visits of cancer patients exceeded those of the general population, which was consistent with our results ${ }^{25}$. A previous study reported that hospitalisation rates and long-term mortality rates of IPF patients admitted to the ICU were high ${ }^{26}$. Although our results showed that the rates of ER visits, all-cause hospitalisation, and ICU admission were significantly higher among IPF patients with cancer than those without cancer, patients with lung cancer showed poorer survival than the other groups. Previous studies found that the difference between the survival rates of IPF patients with lung cancer and those without cancer was not due to the worsening of pulmonary fibrosis; it was mainly due to lung cancer progression and cancer treatment-related complications ${ }^{8,27}$. Interestingly, our results showed that the rate of hospitalisation for cancerrelated complications was significantly higher in IPF patients with lung cancer than those with extrapulmonary cancer. Additionally, the rate of respiratory hospitalisation was highest among IPF patients with lung cancer. These findings suggest that the higher incidence of cancer-related complications and the accompanying rate of respiratory hospitalisation may account for survival differences among the groups.

Our study has several limitations. Due to the retrospective nature of the study, inadequate or missing data could have affected the outcome. However, we used the death registry from the Korean Ministry of the Interior and Safety. Hence, there were no missing data on the date of death. Our data were obtained from a single hospital, and the sample size was relatively small for evaluating the exact prevalence of each type of cancer in patients with IPF. In addition to the low prevalence of IPF, cancer comorbidity among IPF patients is less common. Therefore, further population-based studies are needed to identify the exact prevalence of various cancers in patients with IPF and their prognostic impact. The cause of death could not be determined in some patients because the records for out-of-hospital deaths were unreliable.

In conclusion, this study revealed that the prevalence of cancer among IPF patients was $24 \%$. The overall rates of ER visits, all-cause hospitalisation, and ICU admission were significantly higher among IPF patients with cancer than those without cancer. However, the rate of hospitalisation for cancer-related complications was significantly higher in IPF patients with lung cancer than in those with extrapulmonary cancer. IPF patients with lung cancer had a worse survival than those with extrapulmonary cancer. Interestingly, IPF patients with early-stage lung cancer or extrapulmonary cancer, as well as those without cancer, had comparable survival rates. From these results, physicians should pay attention to the development and progression of cancer and its prognostic impact in patients with IPF. Further population-based studies are needed to validate these results.

\section{Data availability}

The datasets generated during the current study are available from the corresponding author on request.

Received: 2 February 2020; Accepted: 22 September 2020

Published online: 26 October 2020

\section{References}

1. Raghu, G., Weycker, D., Edelsberg, J., Bradford, W. Z. \& Oster, G. Incidence and prevalence of idiopathic pulmonary fibrosis. Am. J. Respir. Crit. Care Med. 174, 810-816. https://doi.org/10.1164/rccm.200602-163OC (2006).

2. Raghu, G., Amatto, V. C., Behr, J. \& Stowasser, S. Comorbidities in idiopathic pulmonary fibrosis patients: a systematic literature review. Eur. Respir. J. 46, 1113-1130. https://doi.org/10.1183/13993003.02316-2014 (2015).

3. Vancheri, C. Idiopathic pulmonary fibrosis: an altered fibroblast proliferation linked to cancer biology. Proc. Am. Thorac. Soc. 9, 153-157. https://doi.org/10.1513/pats.201203-025AW (2012).

4. Raghu, G. et al. An official ATS/ERS/JRS/ALAT statement: idiopathic pulmonary fibrosis: evidence-based guidelines for diagnosis and management. Am. J. Respir. Crit. Care Med. 183, 788-824. https://doi.org/10.1164/rccm.2009-040GL (2011).

5. Le Jeune, I. et al. The incidence of cancer in patients with idiopathic pulmonary fibrosis and sarcoidosis in the UK. Respir. Med. 101, 2534-2540. https://doi.org/10.1016/j.rmed.2007.07.012 (2007).

6. Kato, E. et al. Incidence and predictive factors of lung cancer in patients with idiopathic pulmonary fibrosis. ERJ Open Res. https ://doi.org/10.1183/23120541.00111-2016 (2018).

7. Hyldgaard, C., Hilberg, O. \& Bendstrup, E. How does comorbidity influence survival in idiopathic pulmonary fibrosis?. Respir. Med. 108, 647-653. https://doi.org/10.1016/j.rmed.2014.01.008 (2014).

8. Tomassetti, S. et al. The impact of lung cancer on survival of idiopathic pulmonary fibrosis. Chest 147, 157-164. https://doi. org/10.1378/chest.14-0359 (2015).

9. American Thoracic, S. \& European Respiratory, S. American Thoracic Society/European Respiratory Society International Multidisciplinary Consensus Classification of the Idiopathic Interstitial Pneumonias. This joint statement of the American Thoracic Society (ATS), and the European Respiratory Society (ERS) was adopted by the ATS board of directors, June 2001 and by the ERS Executive Committee, June 2001. Am. J. Respir. Crit. Care Med. 165, 277-304. https://doi.org/10.1164/ajrccm.165.2.ats01 (2002).

10. Lee, K. J. et al. Prevalence, risk factors and survival of lung cancer in the idiopathic pulmonary fibrosis. Thorac. Cancer 3, 150-155. https://doi.org/10.1111/j.1759-7714.2011.00107.x (2012).

11. Ozawa, Y. et al. Cumulative incidence of and predictive factors for lung cancer in IPF. Respirology 14, 723-728. https://doi.org/10 $.1111 / \mathrm{j} .1440-1843.2009 .01547 . x(2009)$.

12. Agudo, A. et al. Impact of cigarette smoking on cancer risk in the European prospective investigation into cancer and nutrition study. J. Clin. Oncol. 30, 4550-4557. https://doi.org/10.1200/JCO.2011.41.0183 (2012).

13. Mohamed Hoesein, F. A. et al. CT-quantified emphysema in male heavy smokers: association with lung function decline. Thorax 66, 782-787. https://doi.org/10.1136/thx.2010.145995 (2011).

14. Jankowich, M. D. \& Rounds, S. Combined pulmonary fibrosis and emphysema alters physiology but has similar mortality to pulmonary fibrosis without emphysema. Lung 188, 365-373. https://doi.org/10.1007/s00408-010-9251-6 (2010).

15. Li, J. et al. Idiopathic pulmonary fibrosis will increase the risk of lung cancer. Chin. Med. J. (Engl.) 127, 3142-3149 (2014). 
16. JafariNezhad, A. \& YektaKooshali, M. H. Lung cancer in idiopathic pulmonary fibrosis: a systematic review and meta-analysis. PLoS ONE 13, e0202360. https://doi.org/10.1371/journal.pone.0202360 (2018).

17. Kreuter, M. et al. Impact of comorbidities on mortality in patients with idiopathic pulmonary fibrosis. PLoS ONE 11, e0151425. https://doi.org/10.1371/journal.pone.0151425 (2016).

18. Cogliano, V. J. et al. Preventable exposures associated with human cancers. J. Natl. Cancer Inst. 103, 1827-1839. https://doi. org/10.1093/jnci/djr483 (2011).

19. du Bois, R. M. et al. Ascertainment of individual risk of mortality for patients with idiopathic pulmonary fibrosis. Am. J. Respir. Crit. Care Med. 184, 459-466. https://doi.org/10.1164/rccm.201011-1790OC (2011).

20. Mura, M. et al. Predicting survival in newly diagnosed idiopathic pulmonary fibrosis: a 3-year prospective study. Eur. Respir. J. 40, 101-109. https://doi.org/10.1183/09031936.00106011 (2012).

21. Ley, B. et al. A multidimensional index and staging system for idiopathic pulmonary fibrosis. Ann. Intern. Med. 156, 684-691. https://doi.org/10.7326/0003-4819-156-10-201205150-00004 (2012).

22. Alakhras, M., Decker, P. A., Nadrous, H. F., Collazo-Clavell, M. \& Ryu, J. H. Body mass index and mortality in patients with idiopathic pulmonary fibrosis. Chest 131, 1448-1453. https://doi.org/10.1378/chest.06-2784 (2007).

23. Ley, B. et al. Idiopathic pulmonary fibrosis: CT and risk of death. Radiology 273, 570-579. https://doi.org/10.1148/radiol.14130 216 (2014).

24. Han, S. et al. Prognosis of non-small-cell lung cancer in patients with idiopathic pulmonary fibrosis. Sci. Rep. 9, 12561. https:// doi.org/10.1038/s41598-019-49026-y (2019).

25. Lash, R. S. et al. A systematic review of emergency department use among cancer patients. Cancer Nurs. 40, 135-144. https://doi. org/10.1097/NCC.0000000000000360 (2017).

26. Saydain, G. et al. Outcome of patients with idiopathic pulmonary fibrosis admitted to the intensive care unit. Am. J. Respir. Crit. Care Med. 166, 839-842. https://doi.org/10.1164/rccm.2104038 (2002).

27. Kreuter, M. et al. Treatment and outcome of lung cancer in idiopathic interstitial pneumonias. Sarcoidosis Vasc. Diffuse Lung Dis. 31, 266-274 (2015).

\section{Author contributions}

S.M.C. and H.Y.L. had full access to all the data in the study, and they take responsibility for the integrity of the data and the accuracy of the data analysis. S.M.C. supervised the conduct of the study. H.Y.L. designed the study, carried out the data analysis, and drafted the initial manuscript. J.C., N.K., J.L., Y.S.P., C.-H.L., S.-M.L., C.-G.Y., Y.W.K., and S.M.C. contributed to the study design and data interpretation and provided critical review and revision of the manuscript for intellectual content. All the authors approved the final manuscript as submitted.

\section{Competing interests}

The authors declare no competing interests.

\section{Additional information}

Supplementary information is available for this paper at https://doi.org/10.1038/s41598-020-75276-2.

Correspondence and requests for materials should be addressed to S.M.C.

Reprints and permissions information is available at www.nature.com/reprints.

Publisher's note Springer Nature remains neutral with regard to jurisdictional claims in published maps and institutional affiliations.

(c) (i) Open Access This article is licensed under a Creative Commons Attribution 4.0 International License, which permits use, sharing, adaptation, distribution and reproduction in any medium or format, as long as you give appropriate credit to the original author(s) and the source, provide a link to the Creative Commons licence, and indicate if changes were made. The images or other third party material in this article are included in the article's Creative Commons licence, unless indicated otherwise in a credit line to the material. If material is not included in the article's Creative Commons licence and your intended use is not permitted by statutory regulation or exceeds the permitted use, you will need to obtain permission directly from the copyright holder. To view a copy of this licence, visit http://creativecommons.org/licenses/by/4.0/.

(C) The Author(s) 2020 